\title{
Osteoporosis Therapies: Evidence from Health-Care Databases and Observational Population Studies
}

\author{
Stuart L. Silverman
}

Received: 28 April 2010/Accepted: 24 June 2010/Published online: 20 August 2010

(C) The Author(s) 2010. This article is published with open access at Springerlink.com

\begin{abstract}
Osteoporosis is a well-recognized disease with severe consequences if left untreated. Randomized controlled trials are the most rigorous method for determining the efficacy and safety of therapies. Nevertheless, randomized controlled trials underrepresent the real-world patient population and are costly in both time and money. Modern technology has enabled researchers to use information gathered from large health-care or medical-claims databases to assess the practical utilization of available therapies in appropriate patients. Observational database studies lack randomization but, if carefully designed and successfully completed, can provide valuable information that complements results obtained from randomized controlled trials and extends our knowledge to real-world clinical patients. Randomized controlled trials comparing fracture outcomes among osteoporosis therapies are difficult to perform. In this regard, large observational database studies could be useful in identifying clinically important differences among therapeutic options. Database studies can also provide important information with regard to osteoporosis prevalence, health economics, and compliance and persistence with treatment. This article describes the
\end{abstract}

The author received editorial/writing support in the preparation of this manuscript, funded by Warner Chilcott Company, LLC, and sanofiaventis U.S. Editorial and writing assistance was provided by Excerpta Medica. The author is fully responsible for all content, editorial decisions, and opinions expressed in this manuscript and received no form of compensation related to the development of this manuscript.

S. L. Silverman $(\bowtie)$

Cedars-Sinai Medical Center, Department of Medicine and

Rheumatology, David Geffen School of Medicine at UCLA, OMC Clinical Research Center, 8641 Wilshire Blvd. Suite 301, Beverly Hills, CA 90211, USA

e-mail: stuarts@omcresearch.org strengths and limitations of both randomized controlled trials and observational database studies, discusses considerations for observational study design, and reviews a wealth of information generated by database studies in the field of osteoporosis.

Keywords Osteoporosis - Randomized controlled trial · Observational database study - Osteoporosis treatment . Effectiveness

Osteoporosis is a worldwide health issue that affects both men and women. Current annual rates of osteoporotic fractures in the United States are estimated to be approximately 2 million [1]. If osteoporosis is left untreated, increasing evidence points to a growing risk of fractures over time [2]. The main aim of osteoporosis treatments is to reduce fracture risk, and randomized controlled trials (RCTs) are the gold standard for determining the efficacy and safety of new drugs.

Over the past decade, large patient databases containing demographic, treatment, and outcome information for a variety of medical conditions have become common in Europe and the United States. Osteoporosis-related fractures are commonly recorded among health-care databases $[3,4]$. Observational database studies can generate valuable information regarding the effectiveness (i.e., efficacy in day-to-day clinical practice) and safety of therapeutic interventions. This type of evidence cannot replace RCT data; however, it can expand these data to a more representative population, identify outcomes to be further studied in other RCTs, and examine which patient subsets would benefit from an intervention [5, 6]. Furthermore, these studies can provide insights into osteoporosis prevalence, the socioeconomic costs associated with fracture, 
and compliance and persistence with treatment. Both RCT and observational database study designs are susceptible to particular bias; therefore, thorough analysis of the advantages and limitations associated with each particular study is needed to interpret the totality of evidence.

\section{Strengths and Limitations of RCTs and Observational Database Studies}

RCTs are designed to minimize bias by selecting narrow and well-defined study populations, as equal as possible with respect to characteristics that may impact on the outcome variables. Participants are randomly assigned to treatment and control groups, ensuring that differences observed in clinical outcomes can be genuinely attributed to treatment assignment $[6,7]$. Additionally, the design of RCTs maximizes patient compliance by ensuring accurate and consistent use of treatment medication through strict protocols and close follow-up [7].

The strict patient inclusion and exclusion criteria of RCTs, however, can limit their ability to provide results that apply to patients seen in day-to-day clinical practice. Specific patient groups included for RCTs might not fully represent those clinical populations for whom the tested drug will eventually be used. Dowd et al. [8] tested this hypothesis by evaluating 120 newly diagnosed osteoporotic female patients with regard to their eligibility for four ongoing RCTs. Although physicians determined all of the 120 patients to be candidates for therapy, only $3-21 \%$ were eligible to participate in the individual RCTs. The most common reasons for patient exclusion were comorbid illness (60\%), concomitant medications $(60 \%)$, high disease severity $(19 \%)$, and age (36\%) [8]. In the clinical practice setting, comorbid illness, concomitant medications, and severe disease are realities in the vast majority of patients with osteoporosis. In general, patients included in RCTs tend to be less sick, younger, and more likely compliant with therapy, all of which may overstate the effect of a new drug should it be introduced in the total patient population. As such, it is important to investigate how therapies impact patients in the real world. In addition, as a result of high costs and difficulties in recruiting eligible patients, RCTs often have a short duration and modest sample size. This means they can be underpowered and unable to detect differences in infrequent clinical outcomes that might be captured in a larger patient population [6]. Furthermore, many patients are simply adverse to the prospect of being randomly selected to participate in a trial, and those who do volunteer may be "healthier" at baseline compared with those unwilling to volunteer, resulting in better outcomes or fewer adverse events [9].

Observational database studies are able to use large unselected patient populations, many of which would be excluded from RCTs, and often can capture data for longer periods, therefore evaluating the long-term effects of therapy, such as the occurrence of rare but perhaps important events [10]. Large database studies can often be used to assess different therapies when head-to-head RCTs are unavailable as a result of elevated costs and large numbers of patients required to participate $[7,11,12]$.

In addition to evaluating effectiveness, observational database studies can estimate real-world compliance and persistence with treatment [13, 14]. This is particularly important for outcomes in osteoporosis because it has been shown that treatment discontinuation is most likely to occur within the first year of therapy [3, 4, 15]. Furthermore, database studies can evaluate the rate of fracture incidence in different populations [16], provide insight into secular changes in fracture incidence [17, 18], and assess costs and health-related quality-of-life outcomes associated with osteoporotic fractures [19].

However, the conclusions drawn from observational database studies also have limitations. The absence of randomization may cause differences in baseline characteristics between study populations, which is known as "selection bias" and could, therefore, compromise the validity of the data. If the different characteristics are related to the measured outcomes, they are referred to as "confounders" and lead to difficulties in determining causality of an observed outcome $[6,7,12]$. Confounding factors include severity of the disease/condition, risk factors, and indications for treatment [7]. Although selection bias may be controlled for and reduced, the impact of bias related to possible known and unknown confounding factors that are not contained in the database, such as body mass index, bone mineral density (BMD), and family history of fracture or prior fracture, cannot be estimated $[6,12]$. Other limitations of observational database studies include the possibility of underreporting and incorrect coding and, in some studies, the inability to validate data through chart review [7, 12].

Regarding osteoporosis, an important limitation of databases is that only a minority of vertebral fractures will be captured since databases do not provide information about morphometric vertebral fractures.

\section{Designing Observational Database Studies}

The validity of conclusions drawn from observational data analysis depends on carefully designed evaluation methodology $[7,12,20]$. One should be certain that the database being studied is sufficiently large to yield the statistical power needed to identify clinically meaningful differences in the outcomes and that the outcomes can be determined from the information available in the database. Internal validity should be maximized by using study populations 
that are as similar as possible, and evaluation of possible confounders should always be included [6, 7]. Although selection bias cannot be entirely eliminated, there are analytical methods to control and reduce this problem. Confounding factors should be controlled by using multivariable adjustment, and the significance and validity of outcomes should be verified by performing sensitivity analyses. In addition, propensity score analysis can statistically adjust for possible selection bias [6, 21]. Understanding what information is accessible within a database and what additional variables are excluded is vital in order to recognize the limitations of any observational database study. Furthermore, accuracy and completeness of the information entered in the database are of great importance to allow valid interpretations and comparisons between studies [22].

Comparing Results from RCTs and Observational Database Studies

Similarities between RCT and observational study data have been identified in several studies for a range of treatments, including cardiology and tuberculosis therapies, as well as surgical interventions [23-26]. However, it is not always the case that RCTs and database studies report similar results. Reasons for discrepancies include different patient populations and therapeutic regimens being evaluated, possible selection bias, inadequate statistical power, and differences in patient follow-up [27]. Additionally, deficiencies in clearly stating patient characteristics (e.g., inclusion and exclusion criteria, demographics), details of treatment, assessment of possible confounders, and duration of follow-up can result in data discrepancies between RCTs and database studies [22].

\section{Observational Database Studies in Osteoporosis}

\section{Secular Trends}

Jaglal et al. [17] studied the time trends in BMD testing for osteoporosis, prescriptions for antiresorptive therapies, and fracture incidence in the Ontario Medicare database 1992-2003. This study has provided important information on the impact of management efforts to reduce osteoporotic fractures in a real-world setting. From 1992 to 2001, the number of BMD tests increased 10-fold, and from 1996 to 2003 the number of prescriptions for antiresorptive therapies increased 18 -fold. It was also found that there was a decrease in the incidence of hip and wrist fractures, with a population projection for 2001-2005 suggesting a downward trend in fracture rates [17].
A recent observational study from Switzerland revealed a significant decrease in age-adjusted incidence of osteoporotic fractures in women but not in men [18]. Although observational studies do not provide evidence for causal relationships, these findings suggest the decline in fracture rates might be due to increased osteoporosis awareness and treatment.

Costs of Treatment and Quality-of-Life Associated with Osteoporotic Fracture

The costs of osteoporosis can be astounding. Estimates have suggested that total annual osteoporotic fracture costs in the United States alone are nearly $\$ 17$ billion [1]. Increased osteoporosis diagnosis and treatment can have a considerable impact on health-care management. A population-based analysis estimated that the BMD testing of an additional 1 million older women in the United States would result in the new prescription of an osteoporosis treatment in 440,000 women and prevention of more than 35,000 fractures, allowing for Medicare cost savings of almost $\$ 78$ million [28]. A study in Sweden assessed the costs and quality-of-life associated with vertebral, hip, and Colles fractures 1 year post-fracture, providing information regarding annual inpatient and outpatient costs [19]. It was shown that although hip fractures were still associated with the highest medical costs, vertebral fractures were associated with a lower quality of life than previously estimated [19]. The design of the Swedish study by Borgström et al. [19] was the foundation of an international observational study, the International Costs and Utilities Related to Osteoporotic Fractures Study (ICUROS) [29], which is currently being conducted in order to help understand the global cost implications of osteoporosis.

\section{Compliance and Persistence}

Several large database studies have provided important insight into real-world patient behavior and osteoporosis medication adherence. Downey et al. [3] evaluated data from a large national, managed-care administrative claims database from four different regions in the United States. Twelve-month adherence rates (defined by a medication possession ratio calculated as total days of therapy for medication dispensed/365 days of study follow-up) were found to be $54-61 \%$ for women receiving antiresorptive therapy [3]. Persistence rates (defined as continuous therapy on the same drug for each month over the entire study period) were found to be $16-21 \%$ [3]. Similar rates were observed in another database study of over 12,000 patients who initiated treatment with either alendronate or risedronate [15]. Other studies have suggested that patient preference for therapy might have a beneficial effect on compliant behavior and 
that preference is greatly dependent on drug efficacy and safety [30-32].

Data from the General Practice Research Database in the United Kingdom demonstrated an inverse relation between compliance and risk of osteoporotic and hip/femur fractures [33]. Patients in this study had a $22 \%$ lower risk of hip/ femur fractures during current use of a bisphosphonate compared with periods of past use. Claims data from two large U.S. databases, representing a geographically diverse population, also showed that patients adherent to therapy have lower fracture rates, with a relative risk $(\mathrm{RR})$ reduction of $20-40 \%$ depending on the fracture site [13]. In a database analysis of all bisphosphonate prescriptions written in Belgium, the RR reduction for hip fracture was as much as $60 \%$ (hazard ratio $[\mathrm{HR}]=0.40,95 \%$ confidence interval [CI] 0.36-0.46, $P<0.0001$ ) [34]. For each $1 \%$ decrease of the medication possession ratio, the risk of hip fracture in this study increased by $0.4 \%$. Another claims database analysis of postmenopausal women with osteoporosis reported that patients persistent with therapy were $26 \%$ less likely to have a fracture diagnosis claim than those who were nonpersistent $(\mathrm{HR}=0.74,95 \%$ CI $0.55-0.99$, $P=0.45$ ) [35]. In this regard, observational database studies can help find solutions for enhancing compliance and persistence by providing insight into why patients might not comply or persist with therapy [3, 36, 37].

In recent years, monthly regimens of bisphosphonates have been developed with the aim of increasing compliance and persistence when compared with daily or weekly regimens. A recent primary-care database study conducted in France using 2007 medical claims data examined this hypothesis [38]. Analyses were performed on data from 2,990 women receiving either weekly bisphosphonates (581 alendronate and 1,408 risedronate) or monthly therapy (1,001 ibandronate). Compliance (defined as a medication possession ratio calculated as the duration of all filled prescriptions/follow-up period) and persistence (defined as time from initiation of therapy to discontinuation, with prespecified permissible gaps) were both superior in the monthly treatment group. Twelve-month persistence rates were $47.5 \%$ for monthly ibandronate and $30.4 \%$ for weekly bisphosphonates. Compliance was $84.5 \%$ in the monthly cohort compared with $79.4 \%$ in the weekly cohort. An important strength of this study was its attention to the effects of potential confounds. A propensity score was constructed, including all demographic, clinical, and treatment variables recorded in the database, and used in the multivariate logistic regression analysis. Although patients receiving monthly ibandronate were younger, were less likely to have had an osteoporotic fracture, more frequently received multiple comedications, and were less likely to have rheumatoid arthritis, dosing frequency remained a significant independent determinant of both compliance and persistence after these variables were taken into account. After this statistical adjustment, women from the monthly cohort were $37 \%$ less likely to be nonpersistent and had a $5 \%$ higher mean medication possession ratio than women receiving the weekly dosing regimens. A potential confound that could not be taken into account in the analysis is that the medication (i.e., ibandronate vs. risedronate or alendronate) was confounded with the dosing frequency (i.e., monthly vs. weekly treatment, respectively), making it difficult to attribute superior compliance and persistence to the dosing frequency rather than an unmeasured variable related to the medication itself.

Although this study supported the association between superior compliance and persistence with a lower dosing frequency, another recent study did not support this relationship. Using the IMS longitudinal prescription database, which contains information on prescription drugs and represents $49 \%$ of the total retail pharmacy prescriptions dispensed in the United States, Gold et al. [39] found that compliance, persistence, and cumulative drug availability were not superior for monthly ibandronate compared with weekly risedronate dosing regimens. Compliance (defined as the mean medication possession ratio), persistence (calculated as the days until a gap of $>90$ days between prescriptions occurred), and cumulative drug availability (calculated as the ratio of drug supply and the days between the first fill date and the end of the study) were evaluated over 12 months among three cohorts: the overall sample, patients new to osteoporosis therapy, and patients receiving therapy in the 12 months after initial market availability for each drug. The three cohorts were created as a means of addressing a potential bias which could have been introduced because of differences in when the two therapies were introduced to the market, i.e., differences in the total number of patients on each therapy as well as in the number of patients new to therapy. Patient age and gender were included as covariates in the analyses. As with the previously described study, medication was confounded with dosing frequency.

At 12 months, persistence, compliance, and cumulative drug availability for the overall sample were significantly higher with weekly risedronate compared to monthly ibandronate. There were no significant differences in compliance for patients new to osteoporosis therapy and for patients new to therapy after initial market availability. Persistence was significantly longer for weekly risedronate than for monthly ibandronate for all three cohorts, and cumulative drug availability was also superior for the weekly regimen compared to the monthly dosing regimen for all three cohorts. These data suggest that the effect of factors other than dosing frequency needs to be explored to elucidate differences in compliance and persistence among users of osteoporosis therapy. 
Safety and Tolerability

To date, few clinical studies have been conducted regarding the long-term safety and tolerability of oral bisphosphonates; however, those that have been published indicate that alendronate and risedronate are well tolerated during extended use [40, 41]. Observational databases can be a resource for determining factors influencing tolerability and long-term safety. A recent RCT of postmenopausal women with osteoporosis reported a higher risk of atrial fibrillation in women treated with zoledronic acid than in placebo patients [42]. Since then, case-control studies have been undertaken to assess the association between atrial fibrillation and the use of bisphosphonates for osteoporosis. Although one of these studies reported the use of alendronate to be associated with an increased risk of atrial fibrillation [43], another report found no evidence that either etidronate or alendronate use increased such risk [44]. To minimize the possibility of confounding, both studies adjusted for important risk factors for atrial fibrillation and used stratification by risk factors. Despite these measures, in the study by Heckbert et al. [43] the number of alendronate users was considerably small (case patients $n=47$ and control patients $n=40$ ), whereas the study by Sørensen et al. [44] had a much larger sample size for bisphosphonate exposure (case patients $n=724$ and control patients $n=3,138$ ). It should be taken into account that recording of events such as atrial fibrillation is not always robust in either RCTs or observational databases.

In order to further determine the risk of atrial fibrillation resulting from bisphosphonate use, a prospective database study of over 47,000 patients was conducted [45]. Data from two sources comprised this study: an ongoing registry of patients who underwent coronary angiography and the Intermountain Healthcare health plans database. No increased risk of atrial fibrillation was found in the bisphosphonate-treated group from either database. Patients in both databases using bisphosphonates were found to be older and more likely to have cardiovascular disease however; and the authors posit that this might account for the increased arrhythmia risk reported in other trials.

Recent studies have reported that long-term alendronate use was common among patients with subtrochanteric or proximal diaphyseal femur fractures, which has led to questions about whether these fractures could be the result of excessive suppression of bone turnover. A register-based matched cohort study in patients with prior non-hip fractures was conducted in order to test the hypothesis that the increase in the risk of subsequent atypical femur fractures in patients treated with alendronate exceeded their risk of typical osteoporotic hip fractures [46]. After adjusting for comorbidity and comedications, there were no significant differences in subtrochanteric or diaphyseal fracture between the alendronate and untreated cohorts. Furthermore, in the small number of patients $(n=178)$ who received long-term alendronate treatment ( $>6$ years) and were highly compliant (medication possession ratio $>80 \%$ ), no differences were observed in the ratio between hip and subtrochanteric/diaphyseal femur fractures between the two cohorts. A total of $10 \%$ of fractures in the alendronate cohort were diaphyseal or subtrochanteric vs. $12.5 \%$ in the control cohort. Although these findings support the conclusion that these fractures are likely consequences of osteoporosis, additional research in a larger cohort of long-term bisphosphonate users is warranted. Specifically, the authors note that this study was not able to provide information specific to transverse subtrochanteric fractures, and radiological and clinical review studies may be able to provide useful information in the future. It should also be noted that access to X-rays is not possible in most observational studies.

Recent reports in the United States as well as in Europe and Japan have linked esophageal cancer with oral bisphosphonate use [47]. Some of these patients have had Barrett esophagus, a precursor of esophageal adenocarcinoma. Safety studies are needed to evaluate the link between oral bisphosphonates and esophageal cancer, but at present, patients with Barrett esophagus should not be prescribed oral bisphosphonates.

\section{Comparison of Osteoporosis Treatments}

An observational database study by Watts et al. [48] used a proprietary administrative claims database to identify managed-care members who received a new prescription for risedronate (6-month analysis $n=1,000$ and 12-month analysis $n=652$ ), alendronate (6-month analysis $n=$ 5,307 and 12-month analysis $n=3,716$ ), or nasal calcitonin (6-month analysis $n=774$ and 12-month analysis $n=656$ ). Patient records were analyzed for the incidence of nonvertebral fractures in the first 6 and 12 months following initiation of treatment. Alendronate and risedronate patients were similar with respect to overall health status, whereas nasal-calcitonin patients had more hospitalizations, physician visits, and concomitant medications, and a higher prior fragility fracture rate. Effectiveness assessments were limited to nonvertebral fractures because the incidence of vertebral fractures was not captured in the administrative claims database. After adjusting for parameters such as age, sex, prior fragility fracture, hormone therapy use, and number of concomitant medications, it was shown that 12 months after treatment initiation nonvertebral fracture risk was reduced by $59 \%$ for patients treated with risedronate vs. alendronate users $(\mathrm{RR}=0.41,95 \% \mathrm{CI}$ $0.18-0.94, P=0.04)$ and by $75 \%$ for patients receiving risedronate compared with calcitonin $(\mathrm{RR}=0.25,95 \% \mathrm{CI}$ 
$0.10-0.64, P<0.01)$. These results support and complement findings observed with individual bisphosphonates in independent RCTs (Fig. 1) [48-50]. In a post hoc analysis, risedronate demonstrated a significant reduction in nonvertebral fracture risk at as early as 6 months (66\%, $P=0.048)$, and at 12 months the risk of nonvertebral fracture was reduced by $74 \%(P=0.001)$ [50]. A post hoc analysis from the alendronate trials demonstrated a significant reduction in the risk of nonvertebral fracture $(26 \%$, $P=0.011)$ at 24 months [49]. The RCTs used BMD or fracture history criteria to identify patients with osteoporosis eligible for the study, whereas the observational study included patients receiving new prescriptions for a bisphosphonate or calcitonin without laboratory diagnostic data confirmation. Nevertheless, results from the observational database study were comparable to those demonstrated by each bisphosphonate in their respective clinical trials.

The results reported by Watts et al. [48], however, contrast with findings from a recent observational database study evaluating the relative effectiveness of alendronate, risedronate, raloxifene, and calcitonin in preventing nonvertebral fractures [51]. Alendronate and risedronate recipients were similar with regard to overall health status, and differences between raloxifene or calcitonin users and alendronate recipients were adjusted for. The study demonstrated that within 12 months of treatment initiation the differences in nonvertebral fracture risk between new recipients of risedronate $(n=8,718)$ or raloxifene $(n=5,038)$ and new alendronate users $(n=21,007)$ were small. New recipients of nasal calcitonin $(n=8,372)$ were at higher risk of nonvertebral fracture compared with alendronate recipients (adjusted $\mathrm{HR}=1.40,95 \% \mathrm{CI}$ $1.20-1.63, P<0.001)$. Similar results were reported for the analysis 24 months after treatment initiation [51].

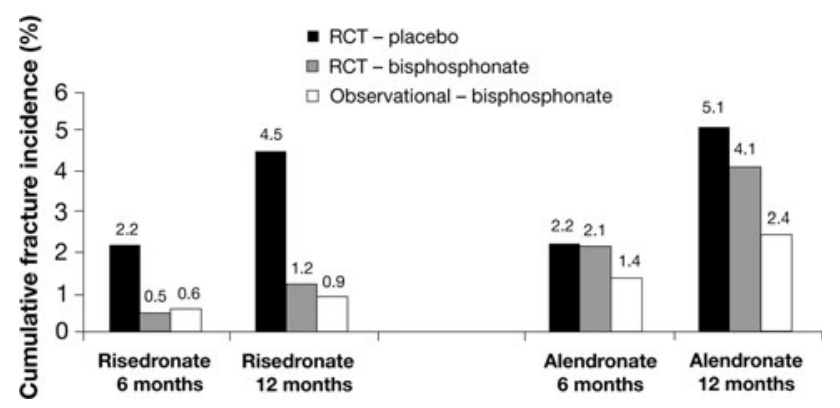

Fig. 1 Nonvertebral fractures in pooled randomized controlled trials (RCTs) and observational database studies. This comparison of previously published data on risk reduction in nonvertebral fractures includes outcomes in RCTs and observational data for two bisphosphonates. Observational data are based on Watts et al. [48]. RCT data are based on Black et al. [49] for alendronate and Harrington et al. [50] for risedronate
Methodological differences in the design of both of these studies, such as the doses of bisphosphonates used and the nonvertebral fracture sites included in the analyses, may partially explain data discrepancies.

\section{Head-to-Head Observational Studies of Approved Bisphosphonates}

The Risedronate and Alendronate (REAL) observational database study [52] recently compared nonvertebral- and hip-fracture risk between two approved bisphosphonates. Although evidence-based consensus guidelines recommend bisphosphonates as first-line treatment for osteoporosis because of their effective prevention of vertebral and, in some cases, nonvertebral fractures, lack of head-to-head comparative trials has precluded the development of recommendations to help select specific individual treatments [53]. A previous head-to-head comparison between alendronate and risedronate used BMD as a surrogate marker of fracture risk prevention, but the study did not consider fracture reduction as an end point [54]. The REAL study has provided an opportunity to compare alendronate and risedronate and evaluate their effectiveness in fracturerisk prevention in a large, clinically relevant patient population.

The REAL study evaluated health-care utilization records from 101 health plans in 34 U.S. states. Subjects included women aged $\geq 65$ years who were newly treated with once-weekly risedronate $(35 \mathrm{mg})$ or alendronate $(35$ or $70 \mathrm{mg}$ ). The 12,215 risedronate users were followed for a mean of 226 days, with $63 \%$ completing 12 months of evaluation. The 21,615 alendronate users $(8 \%$ taking $35 \mathrm{mg}$ and $92 \%$ taking $70 \mathrm{mg}$ ) were followed for a mean of 238 days, with $67 \%$ completing 12 months of evaluation. Statistically significantly, risedronate users were older, used more concomitant medications (including more glucocorticoids), and had comorbid rheumatoid arthritis $(P \leq 0.01)$ compared with alendronate users. This suggests a possible higher pretreatment risk for subsequent fracture among patients treated with risedronate. Conversely, risedronate users had greater previous use of calcitonin or raloxifene, which may contribute to a decreased fracture risk. During the 6- and 12-month historical periods before bisphosphonate initiation, the clinical diagnosis of nonvertebral fracture was similar between subsequent risedronate and alendronate users. However, previous hip fracture had occurred more frequently among patients later prescribed risedronate $(P<0.05)$. This would suggest a higher pretreatment risk for subsequent fracture among patients treated with risedronate.

Cumulative fracture incidence at 6 and 12 months was significantly lower with risedronate compared to alendronate for all nonvertebral and hip fractures $(P \leq 0.05)$ [52]. 
Nonvertebral fracture incidence with risedronate treatment was $19 \%$ lower after 6 months and $18 \%$ lower after 12 months compared with alendronate. Hip-fracture incidence with risedronate treatment was $46 \%$ lower after 6 months and $43 \%$ lower after 12 months compared with alendronate therapy.

One of the strengths of the REAL study was its rigorous sensitivity analysis, which involved an intent-to-treat group analysis, a propensity analysis, a change in inclusion criteria for the study population, and a change in inclusion criteria for the study outcome. Significant differences in nonvertebral- and hip-fracture incidence occurred at 6 months and persisted at 12 months after the various sensitivity analyses. Also, excluding the small minority of patients treated with $35 \mathrm{mg}$ alendronate, outcomes after sensitivity analyses were within the CIs of the primary analyses. Nevertheless, it should be noted that the two patient cohorts were dissimilar with regard to demographics and baseline characteristics, and the effect of these differences in the observed treatment outcomes is unknown.

These data are in agreement with the results reported by Watts et al. [48] and seem to complement previous findings from independent RCTs with each individual bisphosphonate [55-59].

The Risedronate and Alendronate Intervention over Three Years (REALITY) trial used similar methods in a different population to determine differences in clinical fractures between weekly risedronate and alendronate users [60]. This study did not support REAL's findings of significant differences in fracture-risk reduction between the two treatments. Using claims data from a U.S. health-care organization that covered over 20 million people in seven census regions, 19,063 women were identified who were aged $\geq 65$ years and who filled new prescriptions for weekly alendronate $(n=12,956)$ or risedronate $(n=6,107)$. Significantly more risedronate users were aged $\geq 75$ years, used glucocorticoids, had comorbid diabetes, and were more likely to have had a BMD test compared with alendronate users, suggesting a possible higher pretreatment risk for subsequent fracture among patients treated with risedronate. Significant attrition in both cohorts occurred, in large part due to the exclusion of patients for nonadherence (defined by a $>15$-day gap after the end of one prescription before the next prescription was filled). At 1 and 3 years, only $22 \%$ and $3 \%$ of the original cohort remained, respectively.

No significant differences were found between risedronate and alendronate users at 1 year in clinical vertebral or all nonvertebral fracture risk. Although the absolute difference in hip-fracture rate between the two treatments was small (approximately five fractures per 1,000 person-years), the adjusted relative rate of hip fracture among risedronate users was higher $(\mathrm{RR}=1.77,95 \%$ CI $1.15-2.74, P=0.01)$ compared with alendronate users.

Although the patient cohorts in both the REAL and the REALITY studies were similar at baseline, because of the exclusion of substantial numbers of patients due to nonadherence in the REALITY study, patient groups were likely to not be comparable at the time of the 1-year analysis, which could partially explain the discrepant results.

The first head-to-head observational claims database study comparing monthly ibandronate with weekly risedronate and alendronate, the Evaluation of Ibandronate Efficacy (VIBE) study, used eligibility and pharmacy and medical claims data from two large databases comprising almost 90 million people [61]. Women were included in the study if they were aged $\geq 45$ years and were newly prescribed monthly oral ibandronate or weekly oral bisphosphonates (alendronate 35 or $70 \mathrm{mg}$, risedronate $35 \mathrm{mg})$. The primary analysis included 64,182 patients ( $n=7,345$ monthly ibandronate and $n=56,837$ weekly bisphosphonate) adherent for treatment during the first 90 days from the index date (a 30-day refill gap defined nonpersistence for weekly therapy, a 45-day gap was used for monthly therapy). The secondary analysis included all patients $(n=91,598)$ who initiated bisphosphonate treatment regardless of adherence. Sensitivity analyses assessed the effects of potential confounding variables including age; use of other osteoporosis, glucocorticoid, or gastrointestinal medications; and fracture history. Monthly ibandronate patients had significantly more concomitant medications and were more likely to have received gastrointestinal medications and glucocorticoids in the 6-month preindex period.

After the 12-month observational period, the risks of hip, nonvertebral, and any clinical fracture were not significantly different between the two treatment groups (monthly vs. weekly bisphosphonates) among adherent patients. Monthly ibandronate patients had a significantly lower risk of vertebral fracture than weekly bisphosphonate patients (adjusted $\mathrm{RR}=0.36, \quad 95 \%$ CI $0.18-0.75$, $P=0.006)$. When all patients were included in the analysis, the RRs of any type of fracture were not significantly different between the two treatment groups. The results of the sensitivity analyses were generally consistent with the primary analysis.

The 12-month cumulative nonvertebral fracture incidences reported in the REAL, REALITY, and VIBE observational studies are illustrated in Fig. 2.

As fracture reduction is not likely to be immediate following the initiation of bisphosphonate therapy, a limitation common to the REAL, REALITY, and VIBE studies is the uncertainty of whether differences in baseline fracture risk between treatment groups could account for the 


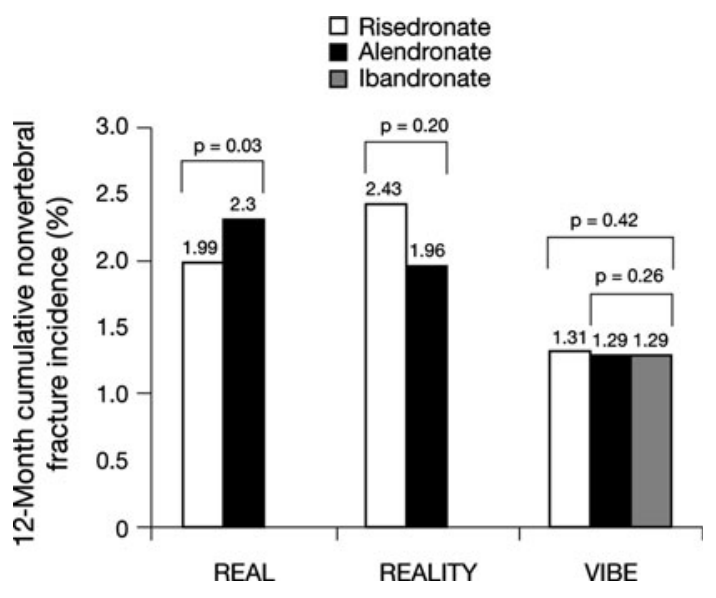

Fig. 2 Cumulative nonvertebral fracture incidence at 12-months. REAL data are based on Silverman et al. [52]. REALITY data are based on Curtis et al. [60]. VIBE data are based on Harris et al. [61]

observed results. A recent observational study addressed this key limitation [62]. Administrative billing data were used to follow three cohorts of U.S. women prescribed alendronate $70 \mathrm{mg} /$ week, risedronate $35 \mathrm{mg} /$ week, or ibandronate $150 \mathrm{mg} / \mathrm{month}$, aged $\geq 65$ years, after initiating either alendronate, risedronate, or ibandronate $(n=$ 210,144). Within each cohort, baseline fracture risk was defined by the initial 3-month period after starting therapy. After 3 months of follow-up, fracture incidence was calculated for the subsequent 12 months of therapy relative to these baselines.

At initiation of bisphosphonate use, the ibandronate cohort was younger and had fewer prior fractures than either the risedronate or alendronate cohort, which suggests a lower pretreatment risk for subsequent fracture. Consistent with these observations, during the 3 months after starting therapy, the baseline incidence of hip fractures was higher in the risedronate $(0.90$ per 100 person-years $)$ and alendronate $(0.77$ per 100 person-years) cohorts than in the ibandronate cohort (0.64 per 100 person-years). When compared to the baseline incidence, the subsequent 12-month fracture incidence was significantly lower in both the alendronate cohort (18\% lower at hip, $28 \%$ at nonvertebral sites, and $57 \%$ at vertebral sites) and the risedronate cohort (27\% lower at hip, $21 \%$ at nonvertebral sites, and $54 \%$ at vertebral sites). Fracture incidence in the ibandronate cohort was $31 \%$ lower at vertebral sites only.

Teriparatide: Effectiveness, Safety, and Persistence

The Direct Analysis of Nonvertebral Fractures in the Community Experience (DANCE) study is an ongoing, prospective, cohort trial that was designed to examine the long-term effectiveness, safety, and tolerability of teriparatide (parathyroid hormone 1-34). The study will also capture data on the reasons for initiating teriparatide therapy and on persistence with treatment [63]. Patients will be followed through a course of therapy for up to 24 months and for an additional 24 months after treatment discontinuation. This study will provide the opportunity to examine the effect of prior and/or concomitant use of other boneactive agents on teriparatide effectiveness at decreasing the occurrence of new nonvertebral fractures. The study population (approximately 4,000 patients) is heterogeneous and includes patients with comorbidities and severe osteoporosis. Data generated by DANCE will be a valuable addition to the already published data on teriparatide. In fact, recent information retrieved from DANCE indicated that the most frequent reasons for physicians to prescribe teriparatide therapy include very low BMD, previous selfreported osteoporotic fracture, general frailty, advanced age, propensity to fall, family history of osteoporotic fractures, and intolerance or inadequate response to other osteoporosis therapies [64]. In addition, it was estimated that approximately $70 \%$ of patients are persistent with teriparatide therapy at 12 months and that persistence was not significantly affected by age, comorbidity, or baseline osteoporosis severity [65]. Nevertheless, the high persistence observed with teriparatide may not be representative of a real-world setting because DANCE has a prospective cohort design, in which the patients are followed through the course of therapy.

\section{Incorporating Observational Data into Clinical Practice}

Observational studies have provided insights into many aspects of osteoporosis and have advanced our knowledge of its epidemiology, patient compliance and persistence with treatment, long-term safety and tolerability of therapies, and costs and quality of life associated with osteoporotic fractures. Observational studies can also provide additional data on the effectiveness of osteoporosis therapies, complementing findings from RCTs and generalizing RCT results to broader patient populations. Analyzing the strengths and limitations associated with each particular study and study type will help to interpret outcomes and their validity. Additional observational database studies comparing treatment effects may be helpful in understanding real-world differences among osteoporosis therapies. Reproducibility of the results, using standardized methodologies, should increase the trust and importance given to data from observational studies.

Acknowledgment Editorial/writing support was funded by Warner Chilcott Company, LLC, and sanofi-aventis U.S. 
Disclosure S. L. S. received research support from Lilly, Merck, Procter \& Gamble, Roche Pharmaceuticals, Roche Diagnostics, Novartis, and Wyeth; is a consultant for Merck, Procter \& Gamble, Roche Pharmaceuticals, Roche Diagnostics, Novartis, and Wyeth; and is part of the speaker's bureau of Lilly, Merck, Procter \& Gamble, and Roche Pharmaceuticals.

Open Access This article is distributed under the terms of the Creative Commons Attribution Noncommercial License which permits any noncommercial use, distribution, and reproduction in any medium, provided the original author(s) and source are credited.

\section{References}

1. Burge R, Dawson-Hughes B, Solomon DH, Wong JB, King A, Tosteson A (2007) Incidence and economic burden of osteoporosis-related fractures in the United States, 2005-2025. J Bone Miner Res 22:465-475

2. Lindsay R, Borisov NN, Sheer RL, Steinbuch M (2005) Impact of early osteoporosis treatment on subsequent fracture prevention among women with clinical vertebral fracture. Presented at: Annual Meeting of the American Society for Bone and Mineral Research, September 23-27, Nashville, TN, abstract M293

3. Downey TW, Foltz SH, Boccuzzi SJ, Omar MA, Kahler KH (2006) Adherence and persistence associated with the pharmacologic treatment of osteoporosis in a managed care setting. South Med J 99:570-575

4. Lo JC, Pressman AR, Omar MA, Ettinger B (2006) Persistence with weekly alendronate therapy among postmenopausal women. Osteoporos Int 17:922-928

5. Stables RH (2002) Observational research in the evidence based environment: eclipsed by the randomised controlled trials? Heart 87:101-102

6. Hannan EL (2008) Randomized clinical trials and observational studies: guidelines for assessing respective strengths and limitations. J Am Coll Cardiol Intv 1:211-217

7. Motheral BR, Fairman KA (1997) The use of claims databases for outcomes research: rationale, challenges, and strategies. Clin Ther 19:346-366

8. Dowd R, Recker RR, Heaney RP (2000) Study subjects and ordinary patients. Osteoporos Int 11:533-536

9. Ward LC, Fielding JW, Dunn JA, Kelly KA (1992) The selection of cases for randomised trials: a registry survey of concurrent trial and non-trial patients. The British Stomach Cancer Group. Br J Cancer 66:943-950

10. Cziraky MJ, Willey VJ, McKenney JM, Kamat SA, Fisher MD, Guyton JR et al (2006) Statin safety: an assessment using an administrative claims database. Am J Cardiol 97:61C-68C

11. Vray M, Hamelin B, Jaillon P; Round Table 2, Giens XX (2005) The respective roles of controlled clinical trials and cohort monitoring studies in the pre- and postmarketing assessment of drugs. Therapie 60:339-344

12. Ayanian JZ (1999) Using administrative data to assess health care outcomes. Eur Heart J 20:1689-1691

13. Siris ES, Harris ST, Rosen CJ, Barr CE, Arvesen JN, Abbott JA et al (2006) Adherence to bisphosphonate therapy and fracture rates in osteoporotic women: relationship to vertebral and nonvertebral fractures from 2 US claims databases. Mayo Clin Proc 81:1013-1022

14. Cramer JA, Gold DT, Silverman SL, Lewiecki EM (2007) A systematic review of persistence and compliance with bisphosphonates for osteoporosis. Osteoporos Int 18:1023-1031
15. Van Staa TP, Geusens P, Leufkens HG, Cooper C (2005) Persistence to bisphosphonate treatment in actual clinical practice. Second joint meeting of the European Calcified Tissue Society and the International Bone and Mineral Society, 25-29 June, Geneva, Switzerland, abstract P610-Su

16. Couris CM, Duclos A, Rabilloud M, Couray-Targe S, Ecochard R, Delmas PD et al (2007) A seventy percent overestimation of the burden of hip fractures in women aged 85 and over. Bone 41:896-900

17. Jaglal SB, Weller I, Mamdani M, Hawker G, Kreder H, Jaakkimainen L et al (2005) Population trends in BMD testing, treatment, and hip and wrist fracture rates: are the hip fracture projections wrong? J Bone Miner Res 20:898-905

18. Chevalley T, Guilley E, Herrmann FR, Hoffmeyer P, Rapin CH, Rizzoli R (2007) Incidence of hip fracture over a 10-year period (1991-2000): reversal of a secular trend. Bone 40:1284-1289

19. Borgström F, Zethraeus N, Johnell O, Lidgren L, Ponzer S, Svensson O et al (2006) Costs and quality of life associated with osteoporosis-related fractures in Sweden. Osteoporos Int 17: $637-650$

20. Black N (1996) Why we need observational studies to evaluate the effectiveness of health care. BMJ 312:1215-1218

21. Lobo FS, Wagner S, Gross CR, Schommer JC (2006) Addressing the issue of channeling bias in observational studies with propensity scores analysis. Res Social Adm Pharm 2:143-151

22. Hartz A, Bentler S, Charlton M, Lanska D, Butani Y, Soomro GM et al (2005) Assessing observational studies of medical treatments. Emerg Themes Epidemiol 2:8

23. Benson K, Hartz AJ (2000) A comparison of observational studies and randomized, controlled trials. N Engl J Med 342:18781886

24. Concato J, Shah N, Horwitz RI (2000) Randomized, controlled trials, observational studies, and the hierarchy of research designs. N Engl J Med 342:1887-1892

25. Wei L, Ebrahim S, Bartlett C, Davey PD, Sullivan FM, McDonald TM (2005) Statin use in the secondary prevention of coronary heart disease in primary care: cohort study and comparison of inclusion and outcome with patients in randomised trials. BMJ 330:821

26. Gross CP, Krumholz HM, Van Wye G, Emanuel EJ, Wendler D (2006) Does random treatment assignment cause harm to research participants? PLoS Med 3:e188

27. King AB, Saag KG, Burge RT, Pisu M, Goel N (2005) Fracture Reduction Affects Medicare Economics (FRAME): impact of increased osteoporosis diagnosis and treatment. Osteoporos Int 16:1545-1557

28. Sørensen HT, Lash TL, Rothman KJ (2006) Beyond randomized controlled trials: a critical comparison of trials with nonrandomized studies. Hepatology 44:1075-1082

29. International Osteoporosis Foundation (2010) International costs and utilities related to osteoporotic fractures study (ICUROS), http://www.iofbonehealth.org/health-professionals/healtheconomics/icuros.html. Cited 23 March 2010

30. Weiss TW, Gold DT, Silverman SL, McHorney CA (2006) An evaluation of patient preferences for osteoporosis medication attributes: results from the PREFER-US study. Curr Med Res Opin 22:949-960

31. Keen R, Jodar E, Iolascon G, Kruse HP, Varbanov A, Mann B et al (2006) European women's preference for osteoporosis treatment: influence of clinical effectiveness and dosing frequency. Curr Med Res Opin 22:2375-2381

32. Gold DT, Safi W, Trinh H (2006) Patient preference and adherence: comparative US studies between two bisphosphonates, weekly risedronate and monthly ibandronate. Curr Med Res Opin 22:2383-2391 
33. Gallagher AM, Rietbrock S, Olson M, van Staa TP (2008) Fracture outcomes related to persistence and compliance with oral bisphosphonates. J Bone Miner Res 23:1569-1575

34. Rabenda V, Mertens R, Fabri V, Vanoverloop J, Sumkay F, Vannecke $\mathrm{C}$ et al (2008) Adherence to bisphosphonates therapy and hip fracture risk in osteoporotic women. Osteoporos Int 19:811-818

35. Gold DT, Martin BC, Frytak JR, Amonkar MM, Cosman F (2007) A claims database analysis of persistence with alendronate therapy and fracture risk in post-menopausal women with osteoporosis. Curr Med Res Opin 23:585-594

36. Silverman S (2006) Adherence to medications for the treatment of osteoporosis. Rheum Dis Clin North Am 32:721-731

37. Gold DT, McClung B (2006) Approaches to patient education: emphasizing the long-term value of compliance and persistence. Am J Med 119(4 Suppl 1):S32-S37

38. Cotte FE, Fardellone P, Mercier F, Gaudin AF, Roux C (2010) Adherence to monthly and weekly oral bisphosphonates in women with osteoporosis. Osteoporos Int 21:145-155

39. Gold DT, Trinh H, Safi W (2009) Weekly versus monthly drug regimens: 1-year compliance and persistence with bisphosphonate therapy. Curr Med Res Opin 25:1831-1839

40. Bone HG, Hosking D, Devogelaer J-P, Tucci JR, Emkey RD, Tonino RP et al (2004) Ten years' experience with alendronate for osteoporosis in postmenopausal women. $\mathrm{N}$ Engl $\mathrm{J}$ Med 350:1189-1199

41. Mellström DD, Sörensen OH, Goemaere S, Roux C, Johnson TD, Chines AA (2004) Seven years of treatment with risedronate in women with postmenopausal osteoporosis. Calcif Tissue Int 75:462-468

42. Black DM, Delmas PD, Eastell R, Reid IR, Boonen S, Cauley JA et al (2007) Once-yearly zoledronic acid for treatment of postmenopausal osteoporosis. N Engl J Med 356:1809-1822

43. Heckbert SR, Li G, Cummings SR, Smith NL, Psaty BM (2008) Use of alendronate and risk of incident atrial fibrillation in women. Arch Intern Med 168:826-831

44. Sørensen HT, Christensen S, Mehnert F, Pedersen L, Chapurlat RD, Cummings SR et al (2008) Use of bisphosphonates among women and risk of atrial fibrilation and flutter: population base case-control study. BMJ 336:813-816

45. Bunch TJ, Anderson JL, May HT, Muhlestein JB, Horne BD, Crandall BG et al (2009) Relation of bisphosphonate therapies and risk of developing atrial fibrillation. Am J Cardiol $103: 824-828$

46. Abrahamsen B, Eiken P, Eastell R (2009) Subtrochanteric and diaphyseal femur fractures in patients treated with alendronate: a register-based national cohort study. J Bone Miner Res 24: 1095-1102

47. Wysowski DK (2009) Reports of esophageal cancer with oral bisphosphonate use. N Engl J Med 360:89-90

48. Watts NB, Worley K, Solis A, Doyle J, Sheer R (2004) Comparison of risedronate to alendronate and calcitonin for early reduction of nonvertebral fracture risk: results from a managed care administrative claims database. J Manag Care Pharm 10:142-151

49. Black DM, Thompson DE, Bauer DC, Ensrud K, Musliner T, Hochberg MC et al (2000) Fracture risk reduction with alendronate in women with osteoporosis: the Fracture Intervention Trial. FIT Research Group. J Clin Endocrinol Metab 85:4118-4124

50. Harrington JT, Ste-Marie LG, Brandi ML, Civitelli R, Fardellone $\mathrm{P}$, Grauer A et al (2004) Risedronate rapidly reduces the risk for nonvertebral fractures in women with postmenopausal osteoporosis. Calcif Tissue Int 74:129-135

51. Cadarette SM, Katz JN, Brookhart MA, Sturmer T, Stedman MR, Solomon DH (2008) Relative effectiveness of osteoporosis drugs for preventing nonvertebral fracture. Ann Intern Med 148: 637-646
52. Silverman SL, Watts NB, Delmas PD, Lange JL, Lindsay R (2007) Effectiveness of bisphosphonates on nonvertebral and hip fractures in the first year of therapy: the risedronate and alendronate (REAL) cohort study. Osteoporos Int 18:25-34

53. North American Menopause Society (2006) Management of osteoporosis in postmenopausal women: 2006 position statement of The North American Menopause Society. Menopause 13:340-367

54. Rosen CJ, Hochberg MC, Bonnick SL, McClung M, Miller P, Broy $S$ et al (2005) Treatment with once-weekly alendronate $70 \mathrm{mg}$ compared with once-weekly risedronate $35 \mathrm{mg}$ in women with postmenopausal osteoporosis: a randomized double-blind study. J Bone Miner Res 20:141-151

55. Black DM, Cummings SR, Karpf DB, Cauley JA, Thompson DE, Nevitt MC et al (1996) Randomised trial of effect of alendronate on risk of fracture in women with existing vertebral fractures. Fracture Intervention Trial Research Group. Lancet 348:15351541

56. Liberman UA, Weiss SR, Bröll J, Minne HW, Quan H, Bell NH et al (1995) Effect of oral alendronate on bone mineral density and the incidence of fractures in postmenopausal osteoporosis. The Alendronate Phase III Osteoporosis Treatment Study Group. N Engl J Med 333:1437-1443

57. Cummings SR, Black DM, Thompson DE, Applegate WB, Barrett-Connor E, Musliner TA et al (1998) Effect of alendronate on risk of fracture in women with low bone density but without vertebral fractures: results from the Fracture Intervention Trial. JAMA 280:2077-2082

58. Harris ST, Watts NB, Genant HK, McKeever CD, Hangartner T, Keller M et al (1999) Effects of risedronate treatment on vertebral and nonvertebral fractures in women with postmenopausal osteoporosis: a randomized controlled trial. Vertebral Efficacy with Risedronate Therapy (VERT) Study Group. JAMA 282:13441352

59. Reginster J, Minne HW, Sorensen OH, Hooper M, Roux C, Brandi ML et al (2000) Randomized trial of the effects of risedronate on vertebral fractures in women with established postmenopausal osteoporosis. Vertebral Efficacy with Risedronate Therapy (VERT) Study Group. Osteoporos Int 11:83-91

60. Curtis JR, Westfall AO, Cheng H, Saag KG, Delzell E (2009) Risedronate and Alendronate Intervention over Three Years (REALITY): minimal differences in fracture risk reduction. Osteoporos Int 20:973-978

61. Harris ST, Reginster JY, Harley C, Blumentals WA, Poston SA, Barr CE et al (2009) Risk of fracture in women treated with monthly oral ibandronate or weekly bisphosphonates: the Evaluation of Ibandronate Efficacy (VIBE) database fracture study. Bone 44:758-765

62. Abelson A, Ringe JD, Gold DT, Lange JL, Thomas T (2009) Longitudinal change in clinical fracture incidence after initiation of bisphosphonates. Osteoporos Int. doi:10.1007/s00198-009-1046-3

63. Miller PD, Silverman SL, Gold DT, Taylor KA, Chen P, Wagman RB (2006) Rationale, objectives and design of the Direct Analysis of Nonvertebral Fracture in the Community Experience (DANCE) study. Osteoporos Int 17:85-90

64. Sebba A, Sierra-Zorita R, Miller P, Chen P, Taylor K, Wong M, et al (2007) The Direct Assessment of Non-vertebral Fracture in the Community Experience (DANCE) study: baseline demographics and reasons for initiating teriparatide therapy. Presented at: Annual Meeting of the American Society for Bone and Mineral Research, September 16-19, Honolulu, HI, abstract W303

65. Taylor K, Gold D, Silverman S, Chen P, Wong M, Krohn K (2007) Persistence with teriparatide therapy among participants in the DANCE trial. Presented at: Annual Meeting of the American Society for Bone and Mineral Research, September 16-19, Honolulu, HI, abstract W384 